\title{
Timing of surgery in patients with aneurysmal subarachnoid haemorrhage: rebleeding is still the major cause of poor outcome in neurosurgical units that aim at early surgery
}

\author{
Y B W E M Roos, L F M Beenen, R J M Groen, K W Albrecht, M Vermeulen
}

Department of

Neurology

Y B W E M Roos

$M$ Vermeulen

Department of

Neurosurgery,

Academic Medical

Centre, University of

Amsterdam, The

Netherlands

$\mathrm{K}$ W Albrecht

Department of

Neurosurgery,

Academic Hospital of

the Free University,

Amsterdam, The

Netherlands

LF M Beenen

Department of Neurosurgery of the Slotervaart Municipal

General Hospital,

Amsterdam, The

Netherlands

R J M Groen

Correspondence to: Dr Yvo Roos, Department of Neurology, Academic Medical Centre (AMC),

Meibergdreef 9, $1105 \mathrm{AZ}$

Amsterdam-Zuidoost,

The Netherlands.

Received 8 October 1996 and in revised form

24 April 1997

Accepted 28 April 1997

\begin{abstract}
Objective-To investigate prospectively the proportion of patients actually operated on early in units that aim at surgery in the acute phase of aneurysmal subarachnoid haemorrhage (SAH) and what is the main current determinant of poor outcome.
\end{abstract}

Methods-A prospective analysis of all SAH patients admitted during a one year period at three neurosurgical units that aim at early surgery. The following clinical details were recorded: age, sex, date of SAH, date of admission to the neurosurgical centre, whether a patient was referred by a regional hospital or a general practitioner, Glasgow coma scale and grade of SAH (World Federation of Neurological Surgeons (WFNS) score) on admission at the neurosurgical unit, results of CT and CSF examination, the presence of an aneurysm on angiography, details of treatment with nimodipine or antifibrinolytic agents, and the date of surgery to clip the aneurysm. At follow up at three months, the patients' clinical outcome was determined with the Glasgow outcome scale and in cases of poor outcome the cause for this was recorded.

Results-The proportion of patients that was operated on early-that is, within three days after SAH-was 55\%. Thirty seven of all 102 admitted patients had a poor outcome. Rebleeding and the initial bleeding were the main causes of this in $35 \%$ and $32 \%$ respectively of all patients with poor outcome.

Conclusions-In neurosurgical units with what has been termed "modern management" including early surgery, about half of the patients are operated on early. Rebleeding is still the major cause of poor outcome.

\section{(F Neurol Neurosurg Psychiatry 1997;63:490-493)}

Keywords: aneurysmal subarachnoid haemorrhage; timing of surgery; rebleeding

Rebleeding in the first three weeks after aneurysmal subarachnoid haemorrhage (SAH) is a very frequent and severe complication. Without operative intervention and without antifibrinolytic treatment about $30 \%$ of the patients have a rebleed within one month of the initial SAH. ${ }^{1}$ In a series of patients with a rebleed established by CT about 50\% progressively deteriorated and died from the rebleed and $30 \%$ died from other complications. Only $20 \%$ of the patients with a rebleed survived at three months and most had severe brain damage. ${ }^{2}$ The occurrence of rebleeds can be reduced not only by early surgery to clip the aneurysm (early surgery usually being defined as surgery within three days after SAH) but also by antifibrinolytic treatment in combination with delayed surgery. ${ }^{3-6}$ Antifibrinolytic treatment has been shown to reduce the occurrence of rebleeding by $60 \% .^{5}$ However, this reduced frequency of rebleeds was accompanied by an increase in the occurrence of cerebral ischaemia which offset the beneficial effects of antifibrinolytic drugs. During the 1980s, prevention and treatment of cerebral ischaemia after SAH have considerably improved. Therefore, the results of a study on the effectiveness of antifibrinolytic drugs might now be different. Whether or not this is true is-according to at least some neurosurgeons and neurologists - an irrelevant question as patients with aneurysmal haemorrhages should be operated on immediately to clip the aneurysm..$^{6-9} \mathrm{~A}$ management protocol with early surgery would virtually eradicate the risk of rebleeding and early surgery can now be carried out with a similar mortality and morbidity as delayed surgery. ${ }^{10}$ Therefore the main cause of mortality and morbidity from SAH is currently considered to be cerebral ischaemia, not rebleeding. ${ }^{11}$

In the neurosurgical units in Amsterdam there is consensus that patients should be operated on in the acute phase and that therefore the question of whether antifibrinolytic treatment is beneficial is clinically of no importance as surgery is delayed in very few patients.

The aim of this study was to investigate prospectively what is the proportion of patients operated on early in units that aim at surgery in the acute phase of aneurysmal SAH and what is currently the main determinant of poor outcome.

Patients and methods

The three centres of this study in Amsterdam are all teaching hospitals. Each centre has at least three neurosurgeons who operate on cerebral aneurysms. Patients are referred to 
these neurosurgical units by 12 regional hospitals, all situated less than $85 \mathrm{~km}$ (50 miles) from the closest centre.

Before the study started it was verified that all three participating neurosurgical units adhered to what has been termed "modern management in SAH". This is treatment with the calcium antagonist nimodipine $(2 \mathrm{mg} / \mathrm{h}$ intravenously or $6 \times 60 \mathrm{mg}$ orally), hypervolaemic and hypertensive treatment to prevent delayed cerebral ischaemia, and early surgery to prevent rebleeding. ${ }^{12-14}$

All patients with the diagnosis "aneurysmal SAH" admitted during a one year period starting 1 January 1995 were prospectively studied. The diagnosis was based on clinical signs and symptoms with confirmation of an aneurysmal bleeding pattern on the initial $\mathrm{CT}^{15}$ If the initial CT was negative and subsequent CSF examination positive (xanthochromia confirmed by spectrophotometry) an aneurysm had to be confirmed on angiography before the patient could be included. ${ }^{16}$ Patients with a perimesencephalic bleeding pattern or other non-aneurysmal SAHs on CT were excluded. ${ }^{17}$

The following clinical details were recorded: age, sex, date of SAH, date of admission to neurosurgical centre, whether a patient was referred by a regional hospital or a general practitioner, Glasgow coma scale, and grade of SAH (World Federation of Neurological Surgeons (WFNS) score) on admission at the neurosurgical unit, ${ }^{18}{ }^{19}$ results of CT and CSF examination, the presence of an aneurysm on angiography, details on treatment with nimodipine or antifibrinolytic agents, and the date of surgery to clip the aneurysm.

At three month follow up, the patients' clinical outcome was determined with the Glasgow outcome scale. ${ }^{20}$ Death, persistent vegetative state and severe disability on the Glasgow outcome scale were combined as "poor outcome" whereas moderate disability and good recovery were recorded in the study forms as "good outcome". In patients with poor outcome, the physician or surgeon in charge of the patient was asked to fill in the cause for this poor outcome: initial bleed, rebleeding, cerebral ischaemia, operative complications, or other complications, which had to be specified. In cases of poor outcome caused by rebleeding or cerebral ischaemia, confirmation was sought on CT or at necropsy. In patients with poor outcome caused by rebleeding the actual timing of this rebleeding, suspected clinically or established on CT or at necropsy, was also recorded.

\section{Results}

CLINICAL CHARACTERISTICS AND TIMING OF

TRANSFER OR ADMISSION

During the year starting 1 January 1995, 102 patients with a diagnosis of aneurysmal SAH were admitted to the three neurosurgical centres. Table 1 shows the characteristics of the patients and the timing of admission to a neurosurgical centre. Two thirds of all patients were referred by regional hospitals; the others were directly referred by general practitioners. Of the 102 patients 57 were admitted at the neurosurgical unit the day of the initial SAH
Table 1 Clinical characteristics and timing of admission at the neurosurgical unit

\begin{tabular}{lc}
\hline Patients (n) & 102 \\
M:F & $1: 2.8$ \\
Median age (y) (range) & $49(20-82)$ \\
Admission to neurosurgical unit after SAH: & \\
$\quad$ Median day (range) & $0(0-33)$ \\
Mean (day) & 2 \\
Median Glasgow coma scale at admission to & \\
neurosurgical unit & $14(3-15)$ \\
World Federation of Neurological Surgeons & \\
score (\%): & \\
I & 35 \\
II & 29 \\
III & 3 \\
IV & 20 \\
V & 13 \\
\hline
\end{tabular}

(day 0$), 18$ on the day after (day 1), 10 on day 2 , one on day 3 , one on day 4 , seven between days 5 and 9 , and eight patients 10 days or more after the SAH. Of the 67 patients who were first referred to a regional hospital 28 $(42 \%)$ were transferred the same day as the initial SAH to a neurosurgical unit (day 0), 16 patients $(24 \%)$ were admitted at the neurosurgical unit the day after the SAH (day 1), nine (13\%) were admitted on day 2 , no patient was admitted on day 3 , one $(1 \%)$ on day 4 , five patients $(7 \%)$ were admitted between days 5 and 9 and eight $(12 \%)$ were admitted 10 days or later after the SAH.

\section{INVESTIGATIONS}

Ninety three patients had an aneurysmal bleeding pattern on the initial CT. A positive lumbar puncture with proof of an aneurysm on subsequent angiography confirmed the diagnosis in all but two of the remaining patients. One patient died shortly after admission but was nevertheless included in the study because at postmortem examination an aneurysmal SAH was established. Another patient with a positive lumbar puncture but without angiography who died, was also included because she had a rebleed established by CT, with a bleeding pattern compatible with an aneurysmal bleeding, before the planned angiography.

Of the 93 patients with an aneurysmal bleeding pattern on CT, 84 patients underwent angiography that showed an aneurysm as the cause of bleeding in 76 patients.

In one of the nine patients with an aneurysmal CT pattern but without angiography, an aneurysm was found at postmortem examination. All of the remaining eight patients without angiography had a poor outcome, five because of the initial bleed (Glasgow coma scale 3 at admission in four patients, one patient with Glasgow coma scale 8), two because of rebleedings (Glasgow coma scale $\geqslant 13$ at admission), and one who was in a nursing home before the SAH.

MEDICAL AND SURGICAL MANAGEMENT

Ninety five patients received treatment with the calcium antagonist nimodipine. The remaining patients had not had this treatment because death had seemed imminent on admission. Eight patients were treated with antifibrinolytic drugs.

Aneurysm surgery was performed in 74 of all 102 patients. In 11 of the remaining 28 patients 
Table 2 Timing of surgery in all patients and in patients who were admitted within three days after $S A H$

\begin{tabular}{|c|c|c|c|c|}
\hline \multirow{2}{*}{$\begin{array}{l}\text { Delay in days } \\
\text { between day of } \\
\text { haemorrhage and } \\
\text { day of surgery }\end{array}$} & \multicolumn{2}{|c|}{ Timing of surgery in all patients } & \multicolumn{2}{|c|}{$\begin{array}{l}\text { Timing of surgery in patients who } \\
\text { were admitted within three days after } \\
\text { SAH }\end{array}$} \\
\hline & Patients $(n(\%))$ & Cumulative (\%) & Patients $(n(\%))$ & Cumulative (\%) \\
\hline 0 & $1(1)$ & 1 & $1(2)$ & 2 \\
\hline 1 & $10(14)$ & 15 & $10(16)$ & 18 \\
\hline 2 & $17(23)$ & 38 & $17(28)$ & 46 \\
\hline 3 & $13(18)$ & 55 & $13(21)$ & 67 \\
\hline 4 & $8(11)$ & 66 & $8(13)$ & 80 \\
\hline 5 & $0(0)$ & 66 & $0(0)$ & 80 \\
\hline 6 & $2(3)$ & 69 & $2(3)$ & 84 \\
\hline $7-14$ & $6(8)$ & 77 & $3(5)$ & 89 \\
\hline $15-21$ & $11(15)$ & 92 & $4(7)$ & 95 \\
\hline$>21$ & $6(8)$ & 100 & $3(5)$ & 100 \\
\hline Total & $74(100)$ & 100 & $61(100)$ & 100 \\
\hline
\end{tabular}

Table 3 Outcome and causes of poor outcome

\begin{tabular}{lc}
\hline & $n(\%)$ \\
\hline Poor outcome & $37(36)$ \\
Cause of poor outcome: & $12(32)$ \\
Initial bleeding & $13(35)$ \\
Rebleeding & $5(14)$ \\
Cerebral ischaemia & $6(16)$ \\
Surgical complications & $1(3)$ \\
Other & \\
\hline
\end{tabular}

without surgery, angiography was not carried out, in seven patients because death seemed imminent on admission and in three because of rebleedings resulting in poor outcome before the planned angiography. In one patient angiography and subsequent surgery were not considered because of poor clinical condition before her SAH. In nine patients angiography did disclose an aneurysm, but eight of these patients died before surgery, three because of the initial bleeding and five because of rebleedings. One patient refused to have surgery to clip the aneurysm. Finally, eight patients were not operated on because angiography (and repeat angiography) did not show an aneurysm. Of these eight, two patients had a poor outcome, one because of rebleeding, one because of cerebral ischaemia. The remaining six patients had a Glasgow coma scale $\geqslant 13$ on admission and had good outcome after three months.

Table 2 shows the time of surgery in relation to the day of the initial SAH in all patients. Fifty five per cent of all operated patients had early surgery to clip the aneurysm-that is, surgery within three days after SAH. Because a major reason for delay between the initial SAH and surgery might be late admittance to a neurosurgical unit, table 2 also shows the same timing of surgery results for patients admitted at the neurosurgical unit within three days after SAH; $33 \%$ of these patients were operated on four days or later after the SAH.

OUTCOME

Thirty seven of all 102 patients had a poor outcome. Table 3 shows the causes in these patients. In patients referred to neurosurgical units aiming at early surgery, rebleeding and the initial bleeding are the main causes of poor outcome.

One patient with two angiographically established aneurysms who was not operated on because of her age ( 80 years) recovered completely and was subsequently discharged from the hospital in good health. Shortly after discharge she died from a probable rebleed (not established on CT or necropsy) one month after her initial SAH. In all other patients with rebleeding as the cause of poor outcome, this rebleeding was confirmed on CT or at necropsy. Four of these patients had this rebleeding on the same day as the initial bleeding, three patients rebled two days after the initial SAH, two patients in the second week, one in the third week, and two patients had a rebleed more than three weeks after the initial SAH.

Cerebral ischaemia as the cause of poor outcome was confirmed on CT or at necropsy in all five patients.

\section{Discussion}

This study shows that in neurosurgical units that aim at early surgery to reduce the risk of rebleeding, the actual proportion of patients operated on early - that is, within three days after SAH-is $55 \%$. Of the patients who were admitted within three days after the SAH and had surgery for aneurysm, the proportion of patients with early surgery was $67 \%$. These figures for surgery in the acute phase are lower than we had expected. This cannot be explained completely by a delay in referral from regional hospitals to the neurosurgical units as $80 \%$ of all referred patients were admitted at the neurosurgical unit within two days after the initial SAH.

How do our figures compare with those of the literature? In the population based study on the clinical course of SAH in King County, Washington, Longstreth et al reported that the delay between SAH and surgery was on average 5.1 days but they did not report the proportion of patients who had surgery within three days after the initial SAH. ${ }^{21}$ Kassell et al reported in the cooperative study on tirilazad mesylate that the median delay between SAH and surgery was 39 hours. ${ }^{22}$ They also reported that $5 \%$ of the patients were operated on between day 7 and day 10 but did not report the timing of surgery in about $40 \%$ to $50 \%$ of the patients. In the recent study by Whitfield et $a l$, in which the effects of a "protocol driven management regime including earlier surgery" were investigated, $53 \%$ of the patients operated on underwent early surgery. ${ }^{10}$ Early surgery was defined in that study as surgery within four days of the initial SAH. The proportion of patients that was operated on within four days was $66 \%$ in our study, which shows that the proportion of patients with early surgery is not much different between British and Dutch centres that aim at early surgery.

Another unexpected finding was that rebleeding is still a major cause of mortality and morbidity. Of all admitted patients $36 \%$ had a poor outcome and in $35 \%$ of these patients this was caused by rebleeding. A similar high proportion of poor outcome was caused by the direct effect of the initial bleeding. Cerebral ischaemia was the cause of poor outcome in $14 \%$ of the patients with poor outcome or in $5 \%$ of all admitted patients. That rebleeding is 
still a major cause of poor outcome was also a finding of two recently published population based studies, in King County and in greater Cincinnati. ${ }^{21}{ }^{23}$ In these studies rebleeding was also more important than cerebral ischaemia as a cause of poor outcome although the timing of surgery was early.

We did not prospectively study the factors that caused delay in surgery because we did not expect to find a considerable proportion of patients with delayed surgery. In some patients surgery was delayed because of the clinical condition on admission. The question is whether in these patients early surgery is beneficial or that other measures should be taken to prevent rebleeding, such as antifibrinolytic treatment or endovascular occlusion of the aneurysm. In other patients surgery was delayed for no obvious reason. It might be that logistic factors played a part.

In review articles on the management of $\mathrm{SAH}$ the impression is given that currently cerebral ischaemia and vasospasm are the main causes of morbidity and mortality after SAH. These reviews urge us to direct all efforts at prevention of vasospasm. ${ }^{11}$ This study shows that in neurosurgical units, with what has been termed "modern management", including early surgery, rebleeding still is a far more important cause of poor outcome than cerebral ischaemia. Therefore the management of patients with aneurysmal SAH should be directed at the prevention of rebleeds. Further studies are needed to investigate whether this can be achieved by surgery alone or whether other measures such as antifibrinolytic treatment or endovascular techniques will be necessary as well.

1 Locksley HB. Natural history of subarachnoid hemorrhage, intracranial aneurysms and arteriovenous malformations. $\mathscr{f}$ Neurosurg 1966;25:321-68.

2 Hijdra A, Vermeulen M, van Gijn J, van Crevel H. Rerupture of intracranial aneurysms: a clinicoanatomic Rerupture of intracranial aneury
study. 7 Neurosurg 1987;67:29-33.

3 Kassell NF, Torner JC, Jane JA, Haley EC Jr, Adams HP The international cooperative study on the timing of aneuThe international cooperative study on the timing of aneu-
rysm surgery. Part 2: surgical results. F Neurosurg 1990;73: rysm surg.

4 Ohman J, Heiskanen O. Timing of operation for ruptured supratentorial aneurysms: a prospective randomized study. f Neurosurg 1989;70:55-60.
5 Vermeulen M, Lindsay KW, Murray GD, et al. Antifibrinolytic treatment in subarachnoid hemorrhage. $N$ Engl $\mathcal{F}$ Med 1984;311:432-7.

6 Adams HP Jr. Antifibrinolytics in aneurysmal subarachnoid hemorrhage. Do they have a role? Maybe. Arch Neurol $1987 ; 44: 114-5$.

7 Weir B. Antifibrinolytics in subarachnoid hemorrhage. Do they have a role? No. Arch Neurol 1987;44:116-8.

8 Haley EC Jr, Torner JC, Kassell NF. Antifibrinolytic therapy and cerebral vasospasm. Neurosurg Clin N Am 1990;1:34956.

9 Thomeer RTWM, Taal JCW, Voormolen JHC, Wintzen AR. Aneurysmal bleeding - a plea for early surgery in good-risk patients. Acta Neurochir 1994;128:126-31.

10 Whitfield PC, Moss H, Ohare D, Smielewski P, Pickard JD, Kirkpatrick PJ. An audit of aneurysmal subarachnoid haemorrhage: earlier resuscitation and surgery reduces inpatient stay and deaths from rebleeding. $\mathcal{F}$ Neurol Neurosurg Psychiatry 1996;60:301-6.

11 Kassell NF. The role of vasospasm in overall outcome from aneurysmal subarachnoid hemorrhage. In: Findlay JM, ed. Cerebral vasospasm. Amsterdam: Elsevier, 1993:27-8.

12 Hasan D, Vermeulen M, Wijdicks EFM, Hijdra A, van Gijn $\mathrm{J}$. Effect of fluid intake and antihypertensive treatment on cerebral ischemia after subarachnoid hemorrhage. Stroke 1989;20:1511-5.

13 Pickard JD, Murray GD, Illingworth R, et al. Effect of oral nimodipine on cerebral infarction and outcome after subarachnoid haemorrhage: British aneurysm nimodipine trial. BMF 1989;298:636-42.

14 Mayberg MR, Batjer HH, Dacey R, et al. Guidelines for the management of aneurysmal subarachnoid hemorrhage. A statement for healthcare professionals from a special writing group of the Stroke Council, American Heart Association. Stroke 1994;25:2315-28.

15 Vermeulen $M$, van Gijn J. The diagnosis of subarachnoid haemorrhage. F Neurol Neurosurg Psychiatry 1990;53:36572 .

16 Vermeulen M, Hasan D, Blijenberg BG, Hijdra A, van Gijn J. Xanthochromia after subarachnoid haemorrhage needs no revisitation. F Neurol Neurosurg Psychiatry 1989;52:8268.

17 Rinkel GJ, Wijdicks EF, Hasan D, et al. Outcome in patients with subarachnoid haemorrhage and negative angiography according to pattern of haemorrhage on computed tomography. Lancet 1991;338:964-8.

18 Teasdale G, Jennett B. Assessment of coma and impaired consciousness. A practical scale. Lancet 1974;ii:81-4.

19 Teasdale GM, Drake CG, Hunt W, et al. A universal subarachnoid hemorrhage scale: report of a committee of the World Federation of Neurosurgical Societies. 7 Neurol Neurosurg Psychiatry 1988;51:1457

20 Jennett B, Bond M. Assessment of outcome after severe brain damage. Lancet 1975; i:480-4.

21 Longstreth WT Jr, Nelson LM, Koepsell TD, van Belle G. Clinical course of spontaneous subarachnoid hemorrhage: a population-based study in King County, Washington. Neurology 1993;43:712-8.

22 Kassell NF, Haley EC, Jr, Apperson-Hansen C, Alves WM. Randomized, double-blind, vehicle-controlled trial of tirilazad mesylate in patients with aneurysmal subarachnoid hemorrhage: a cooperative study in Europe, Australia, and New Zealand. 7 Neurosurg 1996;84:221-8.

23 Broderick JP, Brott TG, Duldner JE, Tomsick T, Leach A. Initial and recurrent bleeding are the major causes of death following subarachnoid hemorrhage. Stroke 1994;25:13427. 